\title{
Highly Sensitive and Reproducible SERS Sensor for Biological pH Detection Based on a Uniform Gold Nanorod Array Platform
}

\author{
Liyan $\mathrm{Bi}^{\dagger}{ }^{\dagger}$ Yunqing Wang, ${ }^{\ddagger}$ Ying Yang, ${ }^{\dagger}$ Yuling $\mathrm{Li}^{\S}{ }^{\S}$ Shanshan Mo," Qingyin Zheng, ${ }^{* \dagger}$ \\ and Lingxin Chen $* * ;$
} ${ }^{\dagger}$ Transformative Otology and Neuroscience Center, College of Special Education, ${ }^{\S}$ Department of Pathophysiology, and
${ }^{\|}$Department of Pharmacy, Binzhou Medical University, Yantai 264003, China
${ }^{\ddagger}$ Key Laboratory of Coastal Environmental Processes and Ecological Remediation, Yantai Institute of Coastal Zone Research, Yantai
264003, China

Supporting Information

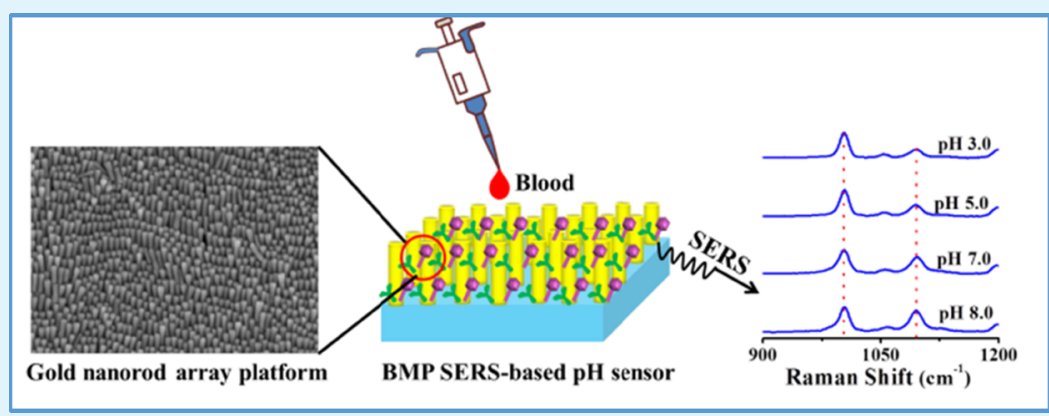

ABSTRACT: Conventional research on surface-enhanced Raman scattering (SERS)-based pH sensors often depends on nanoparticle aggregation, whereas the variability in nanoparticle aggregation gives rise to poor repeatability in the SERS signal. Herein, we fabricated a gold nanorod array platform via an efficient evaporative self-assembly method. The platform exhibits great SERS sensitivity with an enhancement factor of $5.6 \times 10^{7}$ and maintains excellent recyclability and reproducibility with relative standard deviation (RSD) values of less than $8 \%$. On the basis of the platform, we developed a highly sensitive bovine serum albumin (BSA)-coated 4-mercaptopyridine (4-MPy)-linked (BMP) SERS-based $\mathrm{pH}$ sensor to report $\mathrm{pH}$ ranging from $\mathrm{pH}$ 3.0 to $\mathrm{pH}$ 8.0. The intensity ratio variation of 1004 and $1096 \mathrm{~cm}^{-1}$ in 4-MPy showed excellent $\mathrm{pH}$ sensitivity, which decreased as the surrounding $\mathrm{pH}$ increased. Furthermore, this BMP SERS-based $\mathrm{pH}$ sensor was employed to measure the $\mathrm{pH}$ value in C57BL/6 mouse blood. We have demonstrated that the $\mathrm{pH}$ sensor has great advantages such as good stability, reliability, and accuracy, which could be extended for the design of point-of-care devices.

KEYWORDS: gold nanorod array, 4-MPy, SERS, RSD, $p H$ sensor, C57BL/6 mouse blood

\section{INTRODUCTION}

As is well-known, $\mathrm{pH}$ sensing has significant application prospects for bioenvironmental monitoring and biomedical diagnostics. To date, many biosensors have been developed for the $\mathrm{pH}$ probe, such as potentiometric $\mathrm{pH}$ sensors and fluorescent $\mathrm{pH}$ sensors. The potentiometric $\mathrm{pH}$ sensor consists of two types of glass microelectrodes, which suffer from high impedance and fragility. ${ }^{1}$ The fabrication of fluorescent $\mathrm{pH}$ sensors has intrigued interest for high sensitivity as well as for the capability of convenient detection. ${ }^{2,3}$ However, the fluorescent $\mathrm{pH}$ sensor depends on $\mathrm{pH}$-sensitive materials and luminescent probes. As compared to fluorophores, SERS signals are able to resist photobleaching and quenching, display narrower spectral peaks, and be excited with a wide range of wavelengths. ${ }^{4}$ As unique fingerprint spectra, SERS can provide highly resolved vibrational spectroscopic information of analytes even at a femtomolar level, which is extensively used in the biomedical field. ${ }^{5}$
Typically, a SERS-based $\mathrm{pH}$ sensor consists of SERS scaffold and some type of Raman-active $\mathrm{pH}$-probe molecule, ${ }^{6}$ which is responsive to $\mathrm{pH}$ changes. ${ }^{7}$ In general, some weak acids could serve as the $\mathrm{pH}$-probe molecule: 3,5-dimercaptobenzoic acid (DMBA), ${ }^{6}$ 4-mercaptobenzoic acid (MBA), ${ }^{8}$ 4-mercaptopyridine (4-MPy), ${ }^{9}$ aminothiophenol (ATP), ${ }^{10}$ and so on. In past decades, SERS-based $\mathrm{pH}$ sensors have been introduced for imaging $\mathrm{pH}$ distribution and tracing drug release in living cells. Huser and coworkers exhibited that DMBA bound to gold nanoparticles could report $\mathrm{pH}$ distribution in living cells. ${ }^{6}$ Anker and coworkers demonstrated that silica-encapsulated MBA-linked silver nanoparticles could monitor the $\mathrm{pH}$ changes during endocytosis of J774A.1 macrophage cells. ${ }^{11}$ Rector and coworkers fabricated Au@Ag nanoparticles labeled with the pH

Received: December 22, 2017

Accepted: April 17, 2018

Published: April 17, 2018 
probe 4-MPy for SERS-based $\mathrm{pH}$ mapping in rat basophil leukemia cells. ${ }^{12}$ To date, the limitation to SERS-based $\mathrm{pH}$ sensing is that the aggregation of nanoparticles will cause nonuniformity of the SERS signal, which is not amenable to the reliability of the sensors. One strategy against nanoparticle aggregation is to fabricate a highly uniform and reproducible SERS-active platform, in which many developments have been achieved. For instance, nanopillar arrays prepared by laserassisted nanoreplication exhibited high SERS sensitivity. ${ }^{13}$ Ordered $\mathrm{Ag} / \mathrm{SiNW}$ arrays fabricated by nanosphere lithography and sputter deposition showed excellent reproducibility with a RSD of $7-16 \% .^{14}$ Poly(vinyl alcohol) nanofibers decorated with $\mathrm{Ag}$ aggregates via electrospinning displayed superiority of enhancement ability. ${ }^{15}$ All above arrays are restricted by their high cost, complicated steps, and low throughput. ${ }^{16}$ Controlled evaporative self-assembly is a promising approach for tackling the issues, by which we could fabricate a highly uniform, reproducible, and cost-effective SERS-active platform. ${ }^{17}$ Among diverse nanoparticle building blocks, gold nanorods have received increasing attention because of the fact that they exhibit anisotropic optical properties and tunable surface plasmon resonances (SPR). ${ }^{18}$ The characters could offer some significant benefits toward gold nanorod arrays, such as collective plasmon resonances, ${ }^{19}$ strong nanoantenna effect, ${ }^{20}$ and homogeneous "hot spot" surface. ${ }^{16}$

Herein, a novel bovine serum albumin (BSA)-coated 4-MPylinked (BMP) SERS-based $\mathrm{pH}$ sensor was developed for reliable $\mathrm{pH}$ detection, involving a uniform gold nanorod array platform, Raman-active $\mathrm{pH}$-probe molecule, and a protect protein. The schematic diagram of the preparation process employed is given in Figure 1. We focused on the SERS

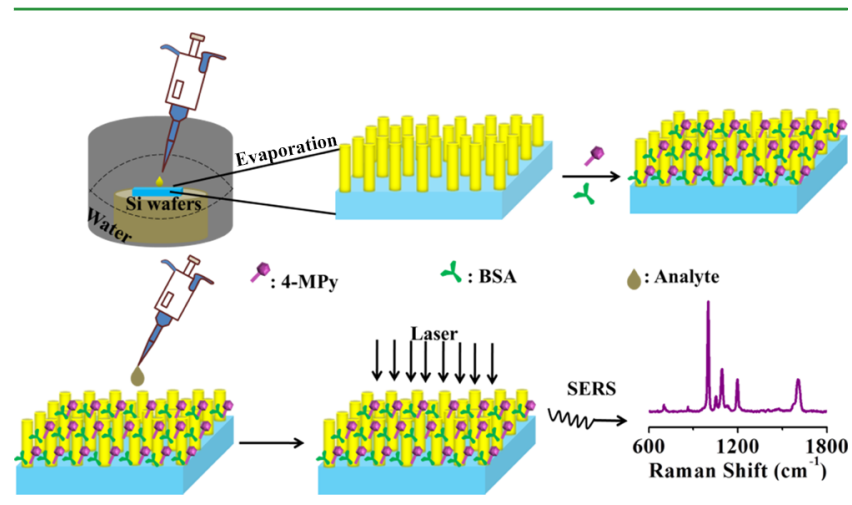

Figure 1. Schematic illustration of the procedure for the preparation of the BMP SERS-based $\mathrm{pH}$ sensor. A drop of gold nanorod suspension on the silicon wafer was kept stationary at room temperature at a humidity of $85 \pm 5 \%$ for up to $72 \mathrm{~h}$ to evaporate the water to obtain vertically aligned gold-nanorod arrays. Then, the gold nanorod array platform was functionalized with 4-MPy and BSA aqueous solution in sequence to obtain the BMP SERS-based $\mathrm{pH}$ sensor.

sensitivity, reproducibility, and recyclability of the gold nanorod array platform. Moreover, the $\mathrm{pH}$ buffer and $\mathrm{pH}$ meter were introduced to investigate the reliability and accuracy, respectively. Finally, we utilized the SERS-based $\mathrm{pH}$ sensor to determine the $\mathrm{pH}$ level in C567/B6 mouse blood. We also believe that the SERS-based $\mathrm{pH}$ sensor could provide a new strategy for the design of point-of-care devices.

\section{EXPERIMENTAL SECTION}

2.1. Materials. Hydrogen tetrachloroauric acid $\left(\mathrm{HAuCl}_{4} \cdot 3 \mathrm{H}_{2} \mathrm{O}\right.$, 99.9\%), cetyltrimethylammonium bromide (CTAB, 99\%), sodium borohydride $\left(\mathrm{NaBH}_{4}\right)$, L-ascorbic acid (AA), and silver nitrate salt $\left(\mathrm{AgNO}_{3}, 99.97 \%\right)$ were all purchased from Sinopharm Chemical Reagent Co., Ltd (Shanghai, China). Hydrochloric acid $(\mathrm{HCl})$ was purchased from Zhongshi Chemical Co., Ltd (Shanghai, China). Citric acid, disodium hydrogen phosphate $\left(\mathrm{Na}_{2} \mathrm{HPO}_{4} \cdot 12 \mathrm{H}_{2} \mathrm{O}\right)$, sodium hydroxide $(\mathrm{NaOH}), 4-\mathrm{MPy}, \mathrm{BSA}$, rhodamine 6G (R6G), and $\mathrm{pH}$ buffer solutions ( $\mathrm{pH}$ 3.0-8.0) were purchased from Aladdin BioChem Technology Co., Ltd (Shanghai, China). The reagents mentioned above were used as received without any treatment. Citric acid phosphate buffer solutions (PBSs) at different $\mathrm{pH}$ values were obtained via mixing $0.1 \mathrm{M}$ citric acid and $0.2 \mathrm{M}$ disodium hydrogen phosphate in appropriate ratios. ${ }^{7}$ The water employed in the study was deionized (resistivity > $18 \mathrm{M} \Omega$ ).

2.2. Experiment Animals. Groups of C57BL/6 mice (female, 710 weeks, $18-20 \mathrm{~g}$ ) were obtained from the animal house at Binzhou Medical University. The animals were kept individually at a temperature of $27 \pm 2{ }^{\circ} \mathrm{C}$ and a humidity of $70-80 \%$ and were subjected to a $12 \mathrm{~h}$ light/dark cycle for $48 \mathrm{~h}$ before use. All of the procedures involving C57BL/6 mice were approved by the Institutional Animal Use and Care Committee of Binzhou Medical University.

2.3. Fabrication of the Gold Nanorod Array Platform. The gold nanorods were synthesized via the previously described seedmediated method in CTAB solutions in the presence of $\mathrm{AgNO}_{3}{ }^{21,22}$ First, the seed was obtained by reduction of $\mathrm{HAuCl}_{4}$ with sodium borohydride. Next, the aged seed solution was subjected to growth solution, involving $\mathrm{CTAB}, \mathrm{HAuCl}_{4}, \mathrm{AgNO}_{3}, \mathrm{AA}$, and an appropriate concentration of $\mathrm{HCl}$. Within $4 \mathrm{~h}$, gold nanorods with an absorption maximum in the visible region were generated after seed addition. The "as-synthesized" gold nanorods were purified by two washing cycles $(8000 \mathrm{rpm}, 30 \mathrm{~min})$ as colloidal suspensions. The concentration of colloidal suspensions was adjusted to $15.0 \mathrm{nM}$, referring to the optical density at $750 \mathrm{~nm}^{23}$ A simple evaporative self-assembly approach was utilized to prepare the gold nanorod array on silicon wafers, which were cleaned with deionized water, acetone, and ethanol in order. As shown in Figure 1, a drop of the colloidal suspensions (15.0 nM, 4.0 $\mu \mathrm{L}$ ) mentioned above was drop-casted on a silicon wafer, which was allowed to dry under standardized environmental conditions $\left(25^{\circ} \mathrm{C}\right.$; $85 \pm 5 \%$ humidity; $72 \mathrm{~h}) .^{24}$ In order to remove the CTAB layer on the obtained slide, pure ethanol was employed to rinse the slide for 5 min, ${ }^{22}$ and the vertically aligned gold nanorod array platform formed.

2.4. Preparation of the BMP SERS-Based $\mathrm{pH}$ Sensor and SERS Measurement. Figure 1 presents the approach for preparing the BMP SERS-based $\mathrm{pH}$ sensor. First, the gold nanorod array platform was functionalized with 4-MPy by immersing it in $0.05 \mathrm{mM}$ 4-MPy for $1 \mathrm{~h}$ to obtain the 4-MPy-linked (MP) SERS-based $\mathrm{pH}$ sensor. Then, the MP SERS-based $\mathrm{pH}$ sensor mentioned above was immersed into $2 \% \mathrm{BSA}$ aqueous solution for $1 \mathrm{~h}$ to obtain the BMP SERS-based $\mathrm{pH}$ sensor. During SERS spectra detection, R6G at different concentrations ranging from $10^{-4}$ to $10^{-9} \mathrm{M}$ was absorbed onto the gold nanorod array platform. Afterward, 50 random spots from five SERS substrates were scanned to evaluate the reproducibility of the proposed platform. Finally, for describing the recyclability, the utilized gold nanorod array platforms were subjected to rinsing for $5 \mathrm{~s}$ and drying under a $\mathrm{N}_{2}$ atmosphere at room temperature. ${ }^{25}$ The $\mathrm{pH}$ dependent SERS experiments were implemented by placing $10 \mu \mathrm{L}$ of citric acid PBS at different $\mathrm{pH}$ values on the prepared $\mathrm{pH}$ sensor. The reliability and accuracy of the BMP SERS-based $\mathrm{pH}$ sensor was confirmed by $\mathrm{pH}$ buffer and $\mathrm{pH}$ meter, respectively. Blood samples were collected from C57BL/6 mice, which took the place of citric acid PBS for $\mathrm{pH}$ analysis.

2.5. Instrument. The as-synthesized gold nanorods were diluted for SPR detection, which was acquired with a Shimadzu UV2600 UVvis-NIR spectrometer. Transmission electron microscopy (TEM) images were determined by JEM-1011 TEM operated at $100 \mathrm{kV}$. The gold nanorod solution was washed with water twice before dropping 
onto the copper grids. Scanning electron microscopy (SEM) images were collected with a Zeiss Ultra Plus SEM at an accelerating voltage of $15 \mathrm{kV}$. SERS measurements were performed using a DXR Raman microscope (Thermo Scientific) with a laser wavelength at $785 \mathrm{~nm}$. All spectra were obtained under the same conditions, in which the power of laser was kept at $1 \mathrm{~mW}$ and the exposure time was $5 \mathrm{~s}$.

\section{RESULTS AND DISCUSSION}

\subsection{Tuning the Longitudinal SPR Peak of the Gold} Nanorods. Among gold nanoparticles, gold nanorods have attracted special attention because of their tunable shapedependent optical characteristics. Gold nanorods have two SPR bands: one is a weak transverse SPR band in the visible region and the other is a strong longitudinal SPR (LSPR) band in the longer wavelength region. ${ }^{26}$ The control of the plasmon peak location was assessed by altering the concentration of $\mathrm{HCl}$, which can effectively retard gold nanorod growth, leading to symmetric, long gold nanorods with a high aspect ratio (AR; the length-to-diameter ratio). In Figure $2 \mathrm{~A}$, we tuned the
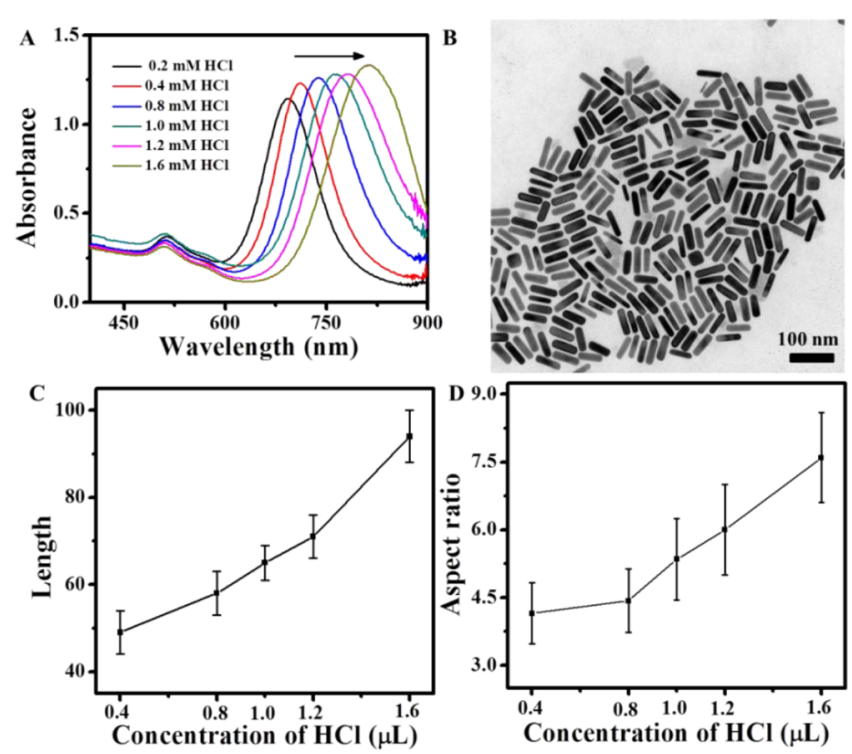

Figure 2. (A) UV-vis-NIR absorption spectra of the synthesized gold nanorods at different concentrations of $\mathrm{HCl}$ ranging from 0.2 to $1.6 \mathrm{mM}$. (B) TEM image of AR $4.4 \pm 0.7$ gold nanorods. (C,D) Corresponding average length and $\mathrm{AR}$ of gold nanorods synthesized under different amounts of $\mathrm{HCl}$. Each data point is presented as mean $\pm \mathrm{SD}$.

distinctive LSPR bands from 690 to $812 \mathrm{~nm}$ and expected gold nanorod to efficiently harvest lights, enhance electric field, and produce a strong SERS signal at a laser line of $785 \mathrm{~nm}$. In Figure 2B, TEM observations on AR $4.4 \pm 0.7$ gold nanorods showed good uniformity, with a length of $65 \pm 4 \mathrm{~nm}$ and LSPR at $785 \mathrm{~nm}$. In Figure 2C,D, as the concentration of $\mathrm{HCl}$ increased from 0.2 to $0.4,0.8,1.0,1.2$, and $1.6 \mathrm{mM}$, the corresponding average length changed from $49 \pm 5$ to $94 \pm 6$ $\mathrm{nm}$ (Figure S1) and the ARs ranged from $4.1 \pm 0.7$ to $7.6 \pm 1.0$ (Table S1). The result is consistent with previous studies. ${ }^{27}$

3.2. Fabrication of the Gold Nanorod Array Platform. Figure 1 exhibited the process for the self-assembly of gold nanorods, in which a $4.0 \mu \mathrm{L}$ gold nanorod suspension was dripped onto a silicon wafer and kept stationary in a highhumidity environment. Such an environment allowed the gold nanorod suspension to evaporate slowly and created enough time to assemble gold nanorods into a vertically aligned gold nanorod array platform, as shown in Figure 3A,B. As the assemblies of nanoparticles are irradiated at the wavelength coupling with the plasmon resonance of the elementary nanoparticles, the junction regions among the adjacent nanoparticles serve as hot spots, and the local electromagnetic fields in the assemblies are dramatically enhanced. ${ }^{5,28,29}$ In this study, the interaction between the exciting line of $785 \mathrm{~nm}$ with AR $4.4 \pm 0.7$ gold nanorod conduction band electrons with the LSPR of $785 \mathrm{~nm}$ could lead to the strongest electromagnetic near fields, which enabled highly sensitive SERS detection. ${ }^{30}$ To examine the sensitivity of gold nanorod arrays, R6G was selected as the label molecule with a series concentration from $10^{-4}$ to $10^{-9} \mathrm{M}$. As shown in Figure 3C, all of the quintessential vibrational modes-including 612, 774, 1362, 1512, and 1651 $\mathrm{cm}^{-1}$-were observed in agreement with the previous report (Table S2). ${ }^{31-34}$ The detection limit for R6G deposited on the gold nanorod array platform was up to $10^{-9} \mathrm{M}$. On this basis, the enhancement factor $(\mathrm{EF})$ values were further calculated to be $\sim 5.6 \times 10^{7}$ (Figure S2). ${ }^{35}$ To evaluate whether the platform features excellent special reproducibility under low concentrations of the analyte, in Figure 3D, we collected Raman waterfall plots of R6G with a concentration of $1 \times 10^{-7} \mathrm{M}$ from the randomly selected positions on the platform. The RSD values were estimated via the previously reported approach. $^{36-38}$ In Figure 3E, the RSD values of signal intensity of R6G at $612,773,1362,1512$, and $1651 \mathrm{~cm}^{-1}$ were calculated to be less than $8 \%$, implying an excellent reproducibility (Table S3). The recyclability property of SERS-active substrates is of crucial importance for the real application. As shown in Figure $3 \mathrm{~F}$, the Raman spectra of $\mathrm{R} 6 \mathrm{G}$ during the recycle process were measured over times, in which R6G could be completely removed via the rinsing process (Figure S4). Furthermore, the SERS intensity at $1512 \mathrm{~cm}^{-1}$ for R6G decreased by less than $10 \%$ over three cycles, revealing that the gold nanorod array platform is a feasible recyclable SERS substrate.

3.3. Fabrication of the BMP SERS-Based pH Sensor. As shown in Figure 1, on the basis of the gold nanorod array platform, 4-MPy was utilized to functionalize the solid surface to gain an MP SERS-based $\mathrm{pH}$ sensor. Then, BSA was anchored onto the MP SERS-based $\mathrm{pH}$ sensor to obtain the BMP SERS-based $\mathrm{pH}$ sensor. 4-MPy serves as a $\mathrm{pH}$-probe molecule, which efficiently binds onto the Au surface via $\mathrm{Au}-\mathrm{S}$ bonds. BSA is chosen as a protective layer, in which disulfide bonds are capable of recognizing the $\mathrm{Au}$ surface to form $\mathrm{Au}-\mathrm{S}$ bonds. The SERS spectra of the MP and BMP SERS-based $\mathrm{pH}$ sensors in the citric acid $\mathrm{PBS}$ of various $\mathrm{pH}$ values ranging from $\mathrm{pH} 3.0$ to 8.0 were shown in Figure 4A,B. The vibrational modes were distinctly visible at $\sim 1004,1061,1096,1208$, and $1607 \mathrm{~cm}^{-1}$ (Table 1). All spectra were normalized against the ring breathing vibration at $\sim 1004 \mathrm{~cm}^{-1}$. No peak position difference between the two $\mathrm{pH}$ sensors mentioned above could be seen, indicating that BSA may not have an impact on the SERS spectra of 4-MPy. Figure 4C showed the zoom-in spectra ranging from 900 to $1200 \mathrm{~cm}^{-1}$ of the BMP SERS-based $\mathrm{pH}$ sensor at different $\mathrm{pH}$ values, in which the signal strength of the ring breathing vibration at $\sim 1004 \mathrm{~cm}^{-1}$ performed in inverse proportion to the $\mathrm{C}-\mathrm{S}$ band at $1096 \mathrm{~cm}^{-1}$ as a function of $\mathrm{pH}$. In Figure 4D, the intensity ratio variation between 1004 and $1096 \mathrm{~cm}^{-1}$ gradually decreased while the $\mathrm{pH}$ value of PBS increased. Herein, we utilized the ratio between the 1004 and $1096 \mathrm{~cm}^{-1}$ bands to monitor the $\mathrm{pH}$ response. The possible model for the resonance structure of 4-MPy could be introduced to account for the remarkable changes in the 

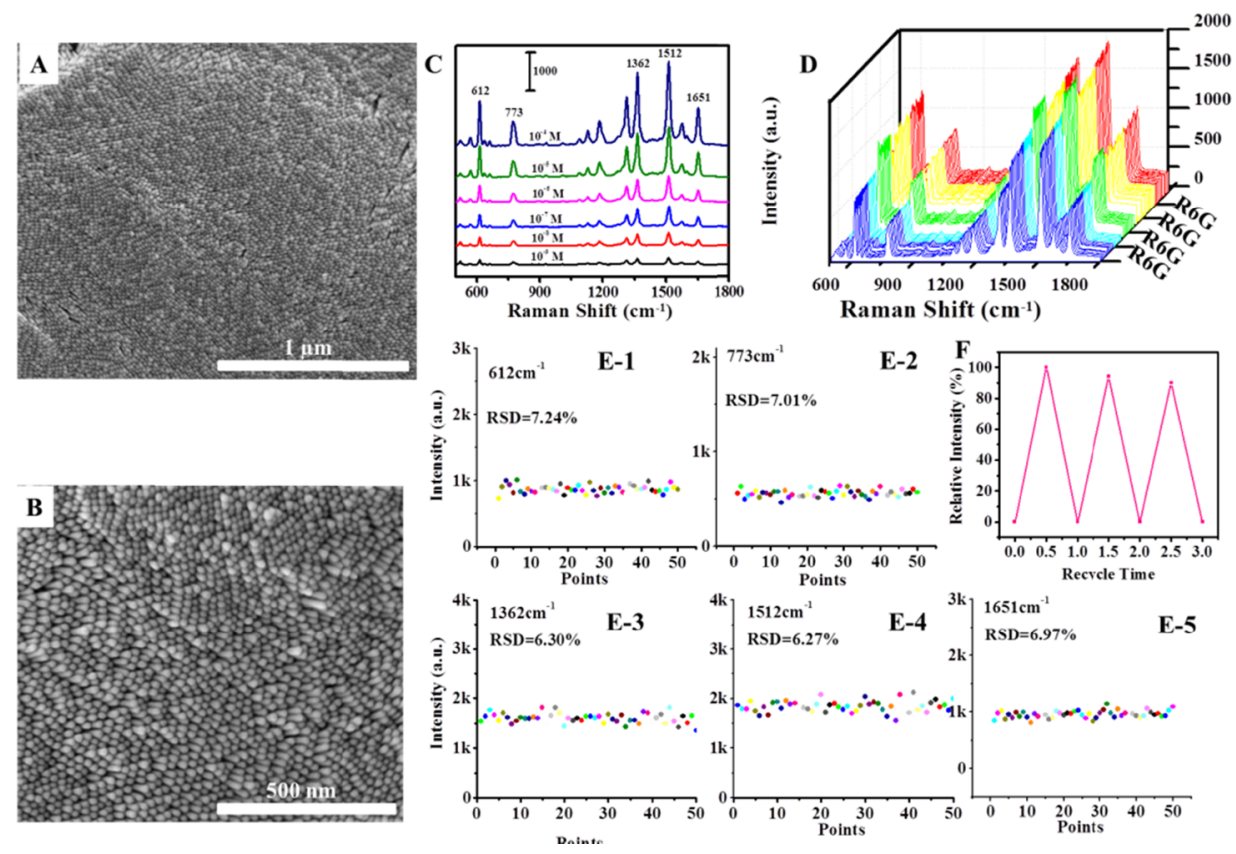

Figure 3. (A,B) SEM images of vertically aligned gold nanorod arrays obtained from drying a gold nanorod suspension on the silicon wafer at different magnifications. (C) SERS spectra of different concentrations of R6G obtained from the gold nanorod array platform. (D) The waterfall plot of SERS spectra (randomly selected areas; the integration time was $2 \mathrm{~s}$ ). (E) RSD of different predominate peaks conveying both intensity and reproducibility of the 50 random points. (F) SERS responses (Raman peak centered at $1512 \mathrm{~cm}^{-1}$ ) of the gold nanorod array platform with R6G and after washing.

Raman spectra. As demonstrated, there are four possibilities for the formation of 4-MPy onto the gold surface, namely, the adsorption through $\mathrm{Au}-\mathrm{S}$ bonds or lone pair electrons of sulfur, ${ }^{39}$ which are responsible for the characteristic changes of the protonation and deprotonation in 4-MPy (Figure S5). At a low $\mathrm{pH}$ value, it tends to form 4- $\mathrm{MPyH}^{+}$, involving mainly one positive charge on a nitrogen atom and aromaticity of the pyridin ring $\left(1004 \mathrm{~cm}^{-1}\right)$. At a high $\mathrm{pH}$ value, it tends to form 4-MPy, including mainly lone pair electrons of nitrogen and $\mathrm{C}=\mathrm{S}$ bond $\left(1096 \mathrm{~cm}^{-1}\right){ }^{40}$ This further illustrated that the signal strength of the ring breathing vibration at $\sim 1004 \mathrm{~cm}^{-1}$ decreased, whereas the signal strength of the $\mathrm{C}-\mathrm{S}$ band at 1096 $\mathrm{cm}^{-1}$ increased along with the $\mathrm{pH}$ level increasing. In Figure $4 \mathrm{E}$, we could see that the intensity ratio between the 1004 and $1096 \mathrm{~cm}^{-1}$ bands in the BMP SERS-based $\mathrm{pH}$ sensor was lower than that in the MP SERS-based $\mathrm{pH}$ sensor. The potential mechanism is that the $\mathrm{Au}-\mathrm{S}$ bonds with the protein (via the 35 Cys residues in BSA) are able to impart good steric protection, ${ }^{41}$ which decreases the ring breathing vibration at $1004 \mathrm{~cm}^{-1}$.

3.4. Characterization of the BMP SERS-Based $\mathrm{pH}$ Sensor. In practical applications, increasing attentions have been paid to the reproducibility and stability of the $\mathrm{pH}$ sensor. Figure 5A exhibited the SERS spectra of the BMP SERS-based $\mathrm{pH}$ sensors from different batches as a function of $\mathrm{pH}$. Compared with the MP SERS-based $\mathrm{pH}$ sensors (Figure S6), the BMP SERS-based $\mathrm{pH}$ sensors showed excellent reproducibility, with small deviations ranging from $\mathrm{pH} 3.0$ to $\mathrm{pH}$ 8.0. In addition, MP SERS-based $\mathrm{pH}$ sensors from different batches showed no regular responses. Figure $5 \mathrm{~B}$ exhibited no obvious change when the temperature ranged from 4 to $37{ }^{\circ} \mathrm{C}$, illustrating that the BMP SERS-based $\mathrm{pH}$ sensors possess good temperature stability. Figure 5C showed the SERS spectra of the BMP SERS-based $\mathrm{pH}$ sensor response to PBS at $\mathrm{pH}$ 3.0, in which the black curve was derived from a freshly prepared $\mathrm{pH}$ sensor and the red curve was derived from a $\mathrm{pH}$ sensor preserved for 7 days. Figure 5D showed the $\mathrm{pH}$ calibration curve before and after preservation. There was no obvious change in peak position and intensity, indicating that the $\mathrm{pH}$ sensor exhibited good stability. More slight variations mentioned above may be a result of the BSA acting as a protective molecule and the gold nanorod array platform acting as a uniform SERS substrate. This indicates that the BMP SERS-based $\mathrm{pH}$ sensors can serve as a good $\mathrm{pH}$ sensor for the investigation of physiological activities.

3.5. $\mathrm{pH}$ Responses Based on the BMP SERS-Based $\mathrm{pH}$ Sensor. To evaluate the accuracy of the $\mathrm{pH}$ sensor, a series of $\mathrm{pH}$ buffer solutions from $\mathrm{pH} 3.0$ to $\mathrm{pH} 8.0$ were drop-casted onto the BMP SERS-based $\mathrm{pH}$ sensor. In Figure 6A, the Raman shift range contained clearly all distinct spectra (Table 1). Figure $6 \mathrm{~B}$ exhibited that the trend of the intensity ratio between 1004 and $1096 \mathrm{~cm}^{-1}$ is consistent with that $\mathrm{pH}$ calibration curve of the BMP SERS-based $\mathrm{pH}$ sensor mentioned above.

Meanwhile, in Figure 6C, the triangle was the $\mathrm{pH}$ meter response to $\mathrm{pH}$ buffer from $\mathrm{pH} 3.0$ to $\mathrm{pH} 8.0$ and the circle was the response of the BMP SERS-based $\mathrm{pH}$ sensor, illustrating that the $\mathrm{pH}$ sensor possessed good accuracy. To demonstrate the potential application of our sensor in biological environment, the BMP SERS-based $\mathrm{pH}$ sensor was employed to determine the $\mathrm{pH}$ value in $\mathrm{C} 57 \mathrm{BL} / 6$ mouse blood. The normal Raman spectrum of blood was used as the control, and no obvious peaks were observed (Figure S7). In Figure 6A, we were able to achieve the intensity ratio between 1004 and 1096 $\mathrm{cm}^{-1}$ of $1.29 \pm 0.002$, which was converted into $\sim \mathrm{pH} 7.45$, as shown in Figure 6D. This study confirms that the SERS-based $\mathrm{pH}$ sensor with the great sensitivity, reliability, and accuracy is promising to serve as the design of point-of-care devices. 

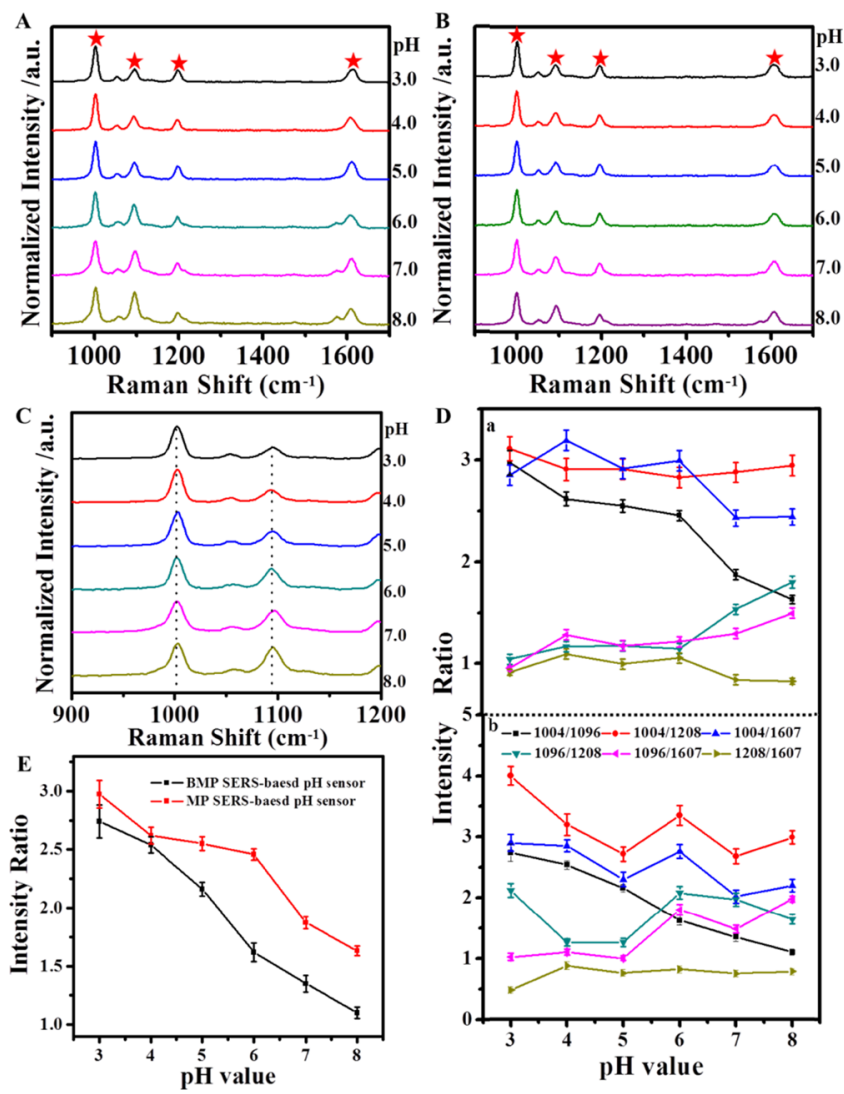

Figure 4. $(\mathrm{A}, \mathrm{B})$ SERS spectra of the BMP SERS-based $\mathrm{pH}$ sensor and MP SERS-based $\mathrm{pH}$ sensor in $\mathrm{PBS}$ of various $\mathrm{pH}$ values ranging from $\mathrm{pH} 3.0$ to $\mathrm{pH} 8.0$ in steps of $1.0 \mathrm{pH}$ unit, respectively. (C) Zoom-in spectra in the spectral range of $900-1200 \mathrm{~cm}^{-1}$ of the BMP SERSbased $\mathrm{pH}$ sensor. (D) Intensity ratio variation of six pairs $\left(1004 \mathrm{~cm}^{-1} /\right.$ $1096 \mathrm{~cm}^{-1}, 1004 \mathrm{~cm}^{-1} / 1208 \mathrm{~cm}^{-1}, 1004 \mathrm{~cm}^{-1} / 1607 \mathrm{~cm}^{-1}, 1096$ $\mathrm{cm}^{-1} / 1208 \mathrm{~cm}^{-1}, 1096 \mathrm{~cm}^{-1} / 1607 \mathrm{~cm}^{-1}$, and $1208 \mathrm{~cm}^{-1} / 1607 \mathrm{~cm}^{-1}$ ) at different $\mathrm{pH}$ values in the MP SERS-based $\mathrm{pH}$ sensor (a) and BMP SERS-based $\mathrm{pH}$ sensor (b), respectively. (E) Intensity ratio variation of $1004 \mathrm{~cm}^{-1} / 1096 \mathrm{~cm}^{-1}$ at different $\mathrm{pH}$ values in the BMP SERSbased $\mathrm{pH}$ sensor and MP SERS-based $\mathrm{pH}$ sensor. Each data point is presented as mean $\pm \mathrm{SD}$.

Table 1. Assignment of the Raman Bands in the Spectra of 4$\mathrm{MPy}$ in Figure $4 \mathrm{~A}^{9}$

\begin{tabular}{ccl}
\multicolumn{2}{c}{ Raman shift $\left(\mathrm{cm}^{-1}\right)$} & \\
\hline experiment & literature & \multicolumn{1}{c}{ assignment } \\
1004 & 1012 & ring breathing \\
1096 & 1093 & X-sensitive/C-S \\
1198 & 1208 & C-H in-plane bend \\
1607 & 1607 & ring stretch \\
\hline
\end{tabular}

\section{CONCLUSIONS}

In summary, we have successfully fabricated a novel BMP SERS-based $\mathrm{pH}$ sensor, involving a 4-MPy and BSA subsequently functionalized gold nanorod array platform. The BMP SERS-based $\mathrm{pH}$ sensor possesses great sensitivity, reliability, and accuracy, which may be a result of the BSA acting as a protective molecule and the gold nanorod array platform acting as a uniform SERS substrate. The gold nanorod array platforms have revealed excellent SERS sensitivity, with $\mathrm{EF}$ values of up to $5.6 \times 10^{7}$. Meanwhile, the gold nanorod array platforms have shown high recyclability and reproduci-
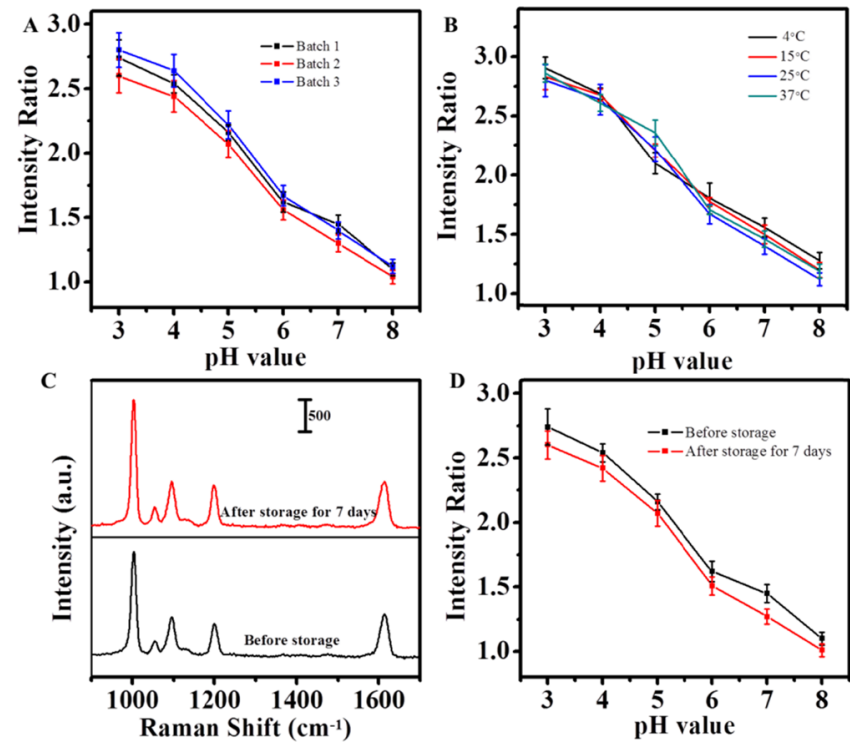

Figure 5. (A) BMP SERS-based $\mathrm{pH}$ sensor from different batches shown with the intensity ratio variation from 1004 to $1096 \mathrm{~cm}^{-1}$ as a function of $\mathrm{pH}$ values. (B) $\mathrm{pH}$ calibration curve of the BMP SERSbased $\mathrm{pH}$ sensor at different temperature $\left(4,15,25\right.$, and $\left.37^{\circ} \mathrm{C}\right)$. (C) SERS spectra of the BMP SERS-based $\mathrm{pH}$ sensor at $\mathrm{pH} 3.0$ before and after storage for 7 days. (D) $\mathrm{pH}$ calibration curve of the BMP SERSbased $\mathrm{pH}$ sensor before and after storage for 7 days. Each data point is presented as mean $\pm \mathrm{SD}$.
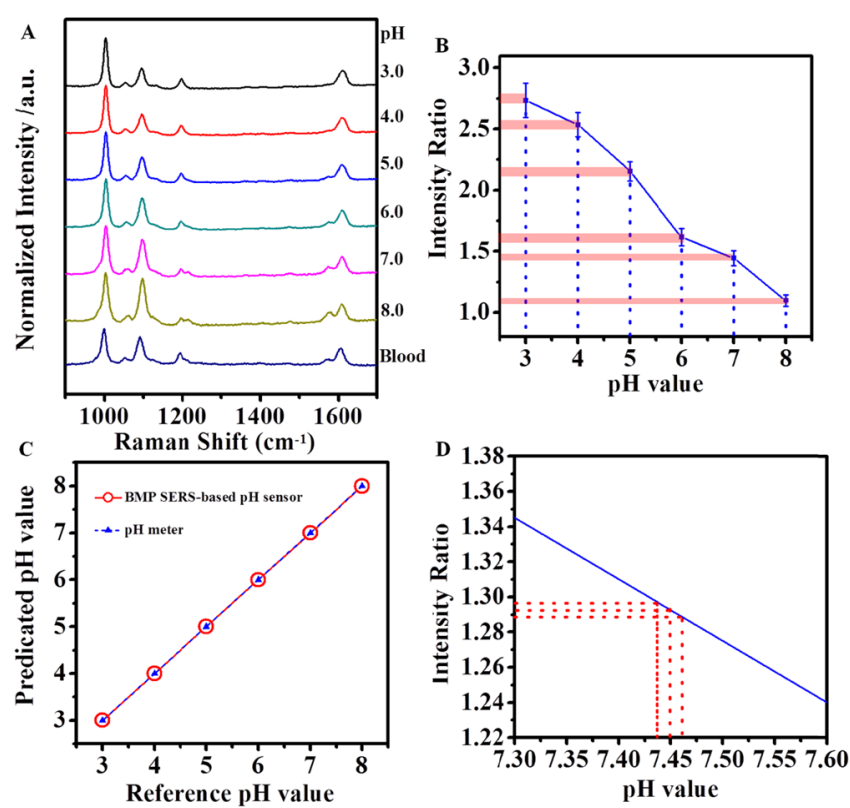

Figure 6. (A) SERS spectra of the BMP SERS-based $\mathrm{pH}$ sensor placed in a series of $\mathrm{pH}$ buffer solutions ranging from $\mathrm{pH} 3.0$ to $\mathrm{pH} 8.0$ and mouse blood. (B) Intensity ratio of $1004-1096 \mathrm{~cm}^{-1}$ obtained from the standard $\mathrm{pH}$ buffer solutions. (C) Standard $\mathrm{pH}$ buffer solutions are measured with the BMP SERS-based $\mathrm{pH}$ sensor and a $\mathrm{pH}$ meter. (D) Measured $\mathrm{pH}$ value of $\mathrm{C} 57 \mathrm{BL} / 6$ mouse blood from the response curve.

bility with an RSD of less than $8 \%$. We also have successfully demonstrated $\mathrm{pH}$ sensing in $\mathrm{C} 57 \mathrm{BL} / 6$ mouse blood. This is one of the few studies to fabricate the $\mathrm{pH}$ sensor based on a planar substrate, which helps to overcome the limitation of nanoparticle aggregation. Furthermore, this study may lead to a 
sensitive biochip platform for the design of point-of-care devices.

\section{ASSOCIATED CONTENT}

\section{S Supporting Information}

The Supporting Information is available free of charge on the ACS Publications website at DOI: 10.1021/acsami.7b19347.

TEM images of gold nanorods; EF calculation process; Raman spectra of R6G; SEM images of vertically aligned gold nanorod array; Raman spectra of R6G at a gold nanorod array platform as a reuse substrate; protonation and deprotonation of resonance structures in 4-MPy; SERS spectra of the MP SERS-based $\mathrm{pH}$ sensor as a function of $\mathrm{pH}$ value; SERS spectra of poly(vinyl pyrrolidone)-, chitosan-, and PBS buffer-coated SERSbased $\mathrm{pH}$ sensors as a function of $\mathrm{pH}$ value; length and diameter of gold nanorods at different concentrations of $\mathrm{HCl}$; assignment of the Raman bands in the spectra of R6G; and RSD values of the SERS substrate from previous work (PDF)

\section{AUTHOR INFORMATION}

\section{Corresponding Authors}

*E-mail: yinqzheng@gmail.com. Phone/Fax: 86-535-6913290, 86-535-2109130 (Q.Z.).

*E-mail: 1xchen@yic.ac.cn (L.C.).

\section{ORCID}

Liyan Bi: 0000-0002-0648-8583

Lingxin Chen: 0000-0002-3764-3515

Notes

The authors declare no competing financial interest.

\section{ACKNOWLEDGMENTS}

We gratefully acknowledge support from the National Natural Science Foundation of China (21605005) and Science and Technology Plan Project of College and University of Shandong Province of China (J16LK10). We also gratefully acknowledge support from the National Natural Science Foundation of China (81530030, 81573393, 21575159, and 51401031) and the Defense Science and Technology Innovation Fund of the Chinese Academy of Sciences (CXJJ17-Q148). At last, the authors would like to express the most sincere thanks to P.H.-S. Jen, Tihua Zheng, and Shuang Shen for continued supports and assistance.

\section{REFERENCES}

(1) Okada, Y.; Inouye, A. pH-Sensitive Glass Microelectrodes and Intracellular pH Measurements. Biophys. Struct. Mech. 1976, 2, 21-30.

(2) Patil, V. S.; Padalkar, V. S.; Phatangare, K. R.; Gupta, V. D.; Umape, P. G.; Sekar, N. Synthesis of New ESIPT-Fluorescein: Photophysics of $\mathrm{pH}$ Sensitivity and Fluorescence. J. Phys. Chem. A 2012, 116, 536-545.

(3) Aguilera-Sigalat, J.; Bradshaw, D. A Colloidal Water-stable MOF as a Broad-range Fluorescent $\mathrm{pH}$ Sensor via Post-synthetic Modification. Chem. Commun. 2014, 50, 4711-4713.

(4) Grubisha, D. S.; Lipert, R. J.; Park, H.-Y.; Driskell, J.; Porter, M. D. Femtomolar Detection of Prostate-specific Antigen: an Immunoassay Based on Surface-Enhanced Raman Scattering and Immunogold Labels. Anal. Chem. 2003, 75, 5936-5943.

(5) Peng, B.; Li, G.; Li, D.; Dodson, S.; Zhang, Q.; Zhang, J.; Lee, Y. H.; Demir, H. V.; Ling, X. Y.; Xiong, Q. Vertically Aligned Gold Nanorod Monolayer on Arbitrary Substrates: Self-Assembly and
Femtomolar Detection of Food Contaminants. ACS Nano 2013, 7, 5993-6000.

(6) Lawson, L. S.; Chan, J. W.; Huser, T. A Highly Sensitive Nanoscale $\mathrm{pH}$-Sensor using Au Nanoparticles Linked by a Multifunctional Raman-active Reporter Molecule. Nanoscale 2014, 6, 79717980.

(7) Zong, S.; Wang, Z.; Yang, J.; Cui, Y. Intracellular pH Sensing using p-Aminothiophenol Functionalized Gold Nanorods with Low Cytotoxicity. Anal. Chem. 2011, 83, 4178-4183.

(8) Talley, C. E.; Jusinski, L.; Hollars, C. W.; Lane, S. M.; Huser, T. Intracellular $\mathrm{pH}$ Sensors Based on Surface-Enhanced Raman Scattering. Anal. Chem. 2004, 76, 7064-7068.

(9) Yu, H.-Z.; Xia, N.; Liu, Z.-F. SERS Titration of 4Mercaptopyridine Self-Assembled Monolayers at Aqueous Buffer/ Gold Interfaces. Anal. Chem. 1999, 71, 1354-1358.

(10) Kong, K. V.; Dinish, U. S.; On Lau, W. K.; Olivo, M. Sensitive SERS-pH Sensing in Biological Media using Metal Carbonyl Functionalized Planar Substrates. Biosens. Bioelectron. 2014, 54, 135140.

(11) Wang, F.; Widejko, R. G.; Yang, Z.; Nguyen, K. T.; Chen, H.; Fernando, L. P.; Christensen, K. A.; Anker, J. N. Surface-Enhanced Raman Scattering Detection of $\mathrm{pH}$ with Silica-encapsulated 4Mercaptobenzoic Acid-Functionalized Silver Nanoparticles. Anal. Chem. 2012, 84, 8013-8019.

(12) Nowak-Lovato, K. L.; Rector, K. D. Targeted Surface-Enhanced Raman Scattering Nanosensors for Whole-cell pH Imagery. Appl. Spectrosc. 2009, 63, 387-395.

(13) Liu, X.; Lebedkin, S.; Besser, H.; Pfleging, W.; Prinz, S.; Wissmann, M.; Schwab, P. M.; Nazarenko, I.; Guttmann, M.; Kappes, M. M.; Lemmer, U. Tailored Surface-Enhanced Raman Nanopillar Arrays Fabricated by Laser-Assisted Replication for Biomolecular Detection using Organic Semiconductor Lasers. ACS Nano 2015, 9, $260-270$

(14) Huang, J.-A.; Zhao, Y.-Q.; Zhang, X.-J.; He, L.-F.; Wong, T.-L.; Chui, Y.-S.; Zhang, W.-J.; Lee, S.-T. Ordered Ag/Si Nanowires Array: Wide-range Surface-Enhanced Raman Spectroscopy for Reproducible Biomolecule Detection. Nano Lett. 2013, 13, 5039-5045.

(15) He, D.; Hu, B.; Yao, Q.-F.; Wang, K.; Yu, S.-H. Large-Scale Synthesis of Flexible Free-Standing SERS Substrates with High Sensitivity: Electrospun PVA Nanofibers Embedded with Controlled Alignment of Silver Nanoparticles. ACS Nano 2009, 3, 3993-4002.

(16) Mei, Z.; Tang, L. Surface-Plasmon-Coupled Fluorescence Enhancement Based on Ordered Gold Nanorod Array Biochip for Ultrasensitive DNA Analysis. Anal. Chem. 2017, 89, 633-639.

(17) Xiao, J.; Li, Z.; Ye, X.; Ma, Y.; Qi, L. Self-Assembly of Gold Nanorods into Vertically Aligned, Rectangular Microplates with a Supercrystalline Structure. Nanoscale 2014, 6, 996-1004.

(18) Edgar, J. A.; McDonagh, A. M.; Cortie, M. B. Formation of Gold Nanorods by a Stochastic "Popcorn" Mechanism. ACS Nano 2012, 6, $1116-1125$.

(19) Hamon, C.; Novikov, S.; Scarabelli, L.; Basabe-Desmonts, L.; Liz-Marzán, L. M. Hierarchical Self-Assembly of Gold Nanoparticles into Patterned Plasmonic Nanostructures. ACS Nano 2014, 8, 1069410703.

(20) Dodson, S. H.; Haggui, M.; Bachelot, R.; Plain, J.; Li, S.; Xiong, Q. Optimizing Electromagnetic Hotspots in Plasmonic Bowtie Nanoantennae. J. Phys. Chem. Lett. 2013, 4, 496-501.

(21) Ming, T.; Feng, W.; Tang, Q.; Wang, F.; Sun, L.; Wang, J.; Yan, C. Growth of Tetrahexahedral Gold Nanocrystals with High-Index Facets. J. Am. Chem. Soc. 2009, 131, 16350-16351.

(22) Zhang, Z.; Yu, Q.; Li, H.; Mustapha, A.; Lin, M. Standing Gold Nanorod Arrays as Reproducible SERS Substrates for Measurement of Pesticides in Apple Juice and Vegetables. J. Food Sci. 2015, 80, N450N458.

(23) Hamon, C.; Postic, M.; Mazari, E.; Bizien, T.; Dupuis, C.; EvenHernandez, P.; Jimenez, A.; Courbin, L.; Gosse, C.; Artzner, F.; Marchi-Artzner, V. Three-Dimensional Self-Assembling of Gold Nanorods with Controlled Macroscopic Shape and Local Smectic B. ACS Nano 2012, 6, 4137-4146. 
(24) Li, P.; Li, Y.; Zhou, Z.-K.; Tang, S.; Yu, X.-F.; Xiao, S.; Wu, Z.; Xiao, Q.; Zhao, Y.; Wang, H.; Chu, P. K. Evaporative Self-Assembly of Gold Nanorods into Macroscopic 3D Plasmonic Superlattice Arrays. Adv. Mater. 2016, 28, 2511-2517.

(25) Mondal, S.; Rana, U.; Malik, S. Facile Decoration of Polyaniline Fiber with Ag Nanoparticles for Recyclable SERS Substrate. ACS Appl. Mater. Interfaces 2015, 7, 10457-10465.

(26) Wang, Y.; Yan, B.; Chen, L. SERS tags: Novel Optical Nanoprobes for Bioanalysis. Chem. Rev. 2013, 113, 1391-1428.

(27) Wang, Y.; Guo, Y.; Shen, Y.; Chen, R.; Wang, F.; Zhou, D.; Zhou, D. HCl-Retarded Gold Nanorod Growth for Aspect Ratio and Shape Tuning. J. Nanosci. Nanotechnol. 2016, 16, 1194-1201.

(28) Thai, T.; Zheng, Y.; Ng, S. H.; Mudie, S.; Altissimo, M.; Bach, U. Self-Assembly of Vertically Aligned Gold Nanorod Arrays on Patterned Substrates. Angew. Chem. 2012, 51, 8732-8735.

(29) Zhu, Z.; Meng, H.; Liu, W.; Liu, X.; Gong, J.; Qiu, X.; Jiang, L.; Wang, D.; Tang, Z. Superstructures and SERS Properties of Gold Nanocrystals with Different Shapes. Angew. Chem., Int. Ed. 2011, 50, $1593-1596$

(30) Han, Z.; Liu, H.; Meng, J.; Yang, L.; Liu, J.; Liu, J. Portable Kit for Identification and Detection of Drugs in Human Urine using Surface-Enhanced Raman Spectroscopy. Anal. Chem. 2015, 87, 95009506.

(31) Zhao, Y.; Yang, D.; Li, X.; Liu, Y.; Hu, X.; Zhou, D.; Lu, Y. Toward Highly Sensitive Surface-Enhanced Raman Scattering: the Design of a 3D Hybrid System with Monolayer Graphene Sandwiched between Silver Nanohole Arrays and Gold Nanoparticles. Nanoscale 2017, 9, 1087-1096.

(32) Jayram, N. D.; Aishwarya, D.; Sonia, S.; Mangalaraj, D.; Kumar, P. S.; Rao, G. M. Analysis on Superhydrophobic Silver Decorated Copper Oxide Nanostructured Thin Films for SERS Studies. J. Colloid Interface Sci. 2016, 477, 209-219.

(33) Li, H.; Wang, Z.; Wang, X.; Jiang, J.; Xu, Y.; Liu, X.; Yan, Y.; Li, C. Preparation of a Self-Cleanable Molecularly Imprinted Sensor based on Surface-Enhanced Raman Spectroscopy for Selective Detection of R6G. Anal. Bioanal. Chem. 2017, 409, 4627-4635.

(34) Tang, W.; Chase, D. B.; Rabolt, J. F. Immobilization of Gold Nanorods onto Electrospun Polycaprolactone Fibers via Polyelectrolyte Decoration-a 3D SERS Substrate. Anal. Chem. 2013, 85, 10702-10709.

(35) Le Ru, E. C.; Blackie, E.; Meyer, M.; Etchegoin, P. G. Surface Enhanced Raman Scattering Enhancement Factors: A Comprehensive Study. J. Phys. Chem. C 2007, 111, 13794-13803.

(36) Zhang, W.; Li, B.; Chen, L.; Wang, Y.; Gao, D.; Ma, X.; Wu, A. Brushing, a Simple Way to Fabricate SERS Active Paper Substrates. Anal. Methods 2014, 6, 2066-2071.

(37) Li, B.; Zhang, W.; Chen, L.; Lin, B. A Fast and Low-cost Spray Method for Prototyping and Depositing Surface-Enhanced Raman Scattering Arrays on Microfluidic Paper Based Device. Electrophoresis 2013, 34, 2162-2168.

(38) Bi, L.; Rao, Y.; Tao, Q.; Dong, J.; Su, T.; Liu, F.; Qian, W. Fabrication of Large-scale Gold Nanoplate Films as Highly Active SERS Substrates for Label-free DNA Detection. Biosens. Bioelectron. 2013, 43, 193-199.

(39) Hu, J.; Zhao, B.; Xu, W.; Li, B.; Fan, Y. Surface-Enhanced Raman Spectroscopy Study on the Structure Changes of 4Mercaptopyridine Adsorbed on Silver Substrates and Silver Colloids. Spectrochim. Acta, Part A 2002, 58, 2827-2834.

(40) Yu, H.-Z.; Xia, N.; Liu, Z. F. SERS Titration of 4Mercaptopyridine Self-Assembled Monolayers at Aqueous Buffer/ Gold Interfaces. Anal. Chem. 1999, 71, 1354-1358.

(41) Xie, J.; Zhang, Q.; Lee, J. Y.; Wang, D. I. C. The Synthesis of SERS-Active Gold Nanoflower Tags for In Vivo Applications. ACS Nano 2008, 2, 2473-2480. 